\title{
Neutrino-nucleon scattering rates in protoneutron stars and nuclear correlations in the spin $S=1$ channel
}

\author{
L. Mornas ${ }^{\mathrm{a}}$ \\ Departamento de Física, Universidad de Oviedo, Avda Calvo Sotelo 18, 33007 Oviedo, Spain
}

Received: 20 June 2005 /

Published online: 27 February 2006 - (c) Società Italiana di Fisica / Springer-Verlag 2006

\begin{abstract}
The neutrino-nucleon cross-section is calculated in dense nuclear matter at finite temperature, in view of applications to supernovae and protoneutron stars. The main contribution to this parameter is provided by the axial response function. Nuclear correlations play an important role: while the $\nu$ - $N$ crosssection is usually reduced by correlations, a collective mode in the spin $S=1$ channel may be excited. In that case, the cross-section diverges and the neutrino mean free path would be drastically reduced. The predictions of various models of the nuclear force commonly used in the literature are compared in relation with a possible spin instability. While Skyrme or Gogny forces lead to attraction in this channel, variational or Brueckner-Hartree-Fock calculations predict a repulsive contribution. An interesting alternative appears to be the density-dependent M3Y interaction.
\end{abstract}

PACS. 26.60.+c Nuclear matter aspects of neutron stars - 25.30.Pt Neutrino scattering - 21.60.Jz HartreeFock and random-phase approximations

\section{Introduction}

With the development of more sensitive neutrino detectors, we would now be able to measure the neutrino burst produced in a supernova explosion with good statistics. At the same time, more powerful computers make it possible to solve numerically the general relativistic collapse together with neutrino transport described by the Boltzmann equations (see, e.g., [1]). The shape of the neutrino burst could thus be predicted - even though this is still obscured in the early stages by the problem of shock stall. Of special interest is the tail of the signal, between 20 and 50 seconds after beginning of the collapse. The outer shells of the supernova should have then been expelled. This corresponds to the deleptonization and first cooling phase of the newly formed protoneutron star. The neutrinos, which were dynamically trapped in the interior by the high density and temperature reached during the collapse, are now gradually released out. During this phase, the neutrino transport can be described by a diffusion equation [2]. The main ingredient of this description, the mean free path, is affected by the state of the matter. Thus, the observation of the tail of the neutrino burst could bring some information on the properties of nuclear matter.

\footnotetext{
${ }^{a}$ e-mail: lysiane@fisi24.ciencias.uniovi.es
}

\section{Neutrino-nucleon scattering rates}

Since the purpose of this work is to investigate the reliability of the available descriptions of the nuclear interactions rather than to make accurate predictions for the neutrino mean free path, a number of simplifications will be made: i) among all possible processes involving neutrinos, only neutrino-nucleon scattering is considered; ii) the interaction potential in the $\mathrm{p}$-h channel will be treated in the Landau-Fermi Liquid approximation. In the non-relativistic approximation, the differential crosssection for $\nu-N$ scattering is given as a function of the energy of the scattered neutrino $E_{\nu}^{\prime}$ and of the scattering angle $\theta$ by

$$
\begin{aligned}
\frac{1}{V} \frac{\mathrm{d} \sigma}{\mathrm{d} \omega \mathrm{d} \Omega}= & \frac{G_{F}^{2}}{8 \pi^{3}}\left(E_{\nu}^{\prime}\right)^{2}\left[1-f\left(E_{\nu}^{\prime}\right)\right] \\
& \times\left[(1+\cos \theta) \mathcal{S}^{(0)}+(3-\cos \theta) \mathcal{S}^{(1)}\right] .
\end{aligned}
$$

In this limit, the spin-0 and spin-1 responses $\mathcal{S}^{(0)}$, $\mathcal{S}^{(1)}$ correspond to the vector and axial couplings, respectively. The former couples to the density correlations/fluctuations while the latter couples to the spindensity correlator:

$$
\left[\begin{array}{l}
\mathcal{S}^{(0)} \\
\mathcal{S}^{(1)}
\end{array}\right]=\left[\begin{array}{l}
c_{V}^{2} \mathcal{S}_{V}(k, \omega) \\
c_{A}^{2} \mathcal{S}_{A}(k, \omega)
\end{array}\right] \sim \int e^{-i \omega t+i k \cdot x}\left[\begin{array}{c}
\langle n(x, t) n(0)\rangle \\
\left\langle j^{i}(x, t) j^{j}(0)\right\rangle
\end{array}\right] .
$$


The main contribution comes from the axial response function, for which reason the neutrino-nucleon mean free path will be specially sensitive to the properties of the nuclear interaction in the spin $S=1$ channel.

\subsection{Medium effects}

It has long been known (see, e.g., [3]) that the neutrinonucleon cross-section is affected by the fact that the nucleons with which it is interacting are not free, but strongly correlated. The medium effects are of various types. A straightforward correction is to treat the nucleons in the mean-field approximation, where they acquire effective masses, momenta and chemical potentials due to their interactions with the other nucleons surrounding them. If one goes further to the random phase approximation (RPA), we are taking into account the screening of the nuclear interaction by particle-hole production. Usually, the RPA correlations are found to reduce the cross-section. If, however, a collective mode is excited, there is a sizable enhancement. For Skyrme parametrizations of the nuclear interaction, for example, a spin instability - which is identified in pure neutron matter with the onset of ferromagnetism - appears at high density. In this case, a pole is present in the cross-section and the mean free path consequently goes to zero $[4,5,6,7]$. Another effect, the broadening of the width of the nucleon, which is important for the Bremsstrahlung and modified URCA processes, will not be treated in this contribution.

The vector and axial structure functions are proportional to the imaginary part of the corresponding polarizations. In the random phase approximation, the polarizations obey Dyson equations:

$$
\Pi_{V / A}^{\mathrm{RPA}}=\Pi_{0}+\Pi_{V / A}^{\mathrm{RPA}} V_{(S=0 / 1)} \Pi_{0} .
$$

In asymmetric $n p$ matter in $\beta$ equilibrium, these equations have a $2 \times 2$ matrix structure, e.g.,

$$
\Pi_{0}=\left[\begin{array}{cc}
\Pi_{n}^{0} & 0 \\
0 & \Pi_{p}^{0}
\end{array}\right] \quad \text { (Linhardt function) }
$$

The interaction potential in the $\mathrm{p}$-h channel in the Landau-Fermi Liquid approximation is

$$
V_{(S=0)}=\left[\begin{array}{ll}
f_{n n} & f_{n p} \\
f_{p n} & f_{p p}
\end{array}\right], \quad V_{(S=1)}=\left[\begin{array}{ll}
g_{n n} & g_{n p} \\
g_{p n} & g_{p p}
\end{array}\right] .
$$

The Landau parameters $f_{i j}, g_{i j}$ describe the interaction between particles having momenta close to the Fermi momentum. They can be obtained, e.g., from the second functional derivative of the polarized energy functional with respect to the density (or from the first derivative of the single-particle potential). In order to keep things as simple as possible we will work in the monopolar approximation $l=0$ (no angular or momentum dependence). In this case, the Landau parameters are real functions of the partial densities of neutrons and protons.

\subsection{Spin (in-)stability}

The criterion for a spin instability to occur is when the determinant of the inverse magnetic susceptibility matrix $\left(\chi_{i j}\right.$, where $\left.i, j \in\{n, p\}\right)$ vanishes. In terms of the Landau parameters:

$$
\operatorname{Det}\left(\frac{1}{\chi_{i j}}\right)=0 \leftrightarrow \operatorname{Det}\left(\begin{array}{cc}
\left(1+G_{0}^{n n}\right) & G_{0}^{n p} \\
G_{0}^{p n} & \left(1+G_{0}^{p p}\right)
\end{array}\right)=0,
$$

where $G_{0}^{i j}=\sqrt{N_{0}^{i} N_{0}^{j}} g_{0}^{i j}$ and $N_{0}^{i}=m_{i}^{*} k_{F i} / \pi^{2}$.

On the other hand, when solving the RPA equations, we obtain, e.g.,

$$
\Pi_{A n n}^{\mathrm{RPA}}=\frac{\left(1-g_{p p} \Pi_{p}^{0}\right) \Pi_{n}^{0}}{\operatorname{Det}\left[I-V_{(S=1)} \Pi_{0}\right]} .
$$

In the limit where the temperature $T$ and the energy transfer $\omega$ go to zero we find

$$
\mathcal{R e} \Pi_{0}^{i}(\omega, k) \rightarrow-N_{0}^{i} \quad \Rightarrow D_{A}=\operatorname{Det}\left[I-V_{(S=1)} \Pi_{0}\right]=0
$$

A spin instability, therefore, gives rise to a peak in the structure function and in the neutrino cross-section. Such a spin instability has been observed to appear in nonrelativistic (e.g. Skyrme) [8] as well as in relativistic $(\sigma \omega \rho)$ models [9] of the nuclear interaction. It has been argued that it could be related to the exchange (Fock) part of the interaction. On the other hand, such an instability is not seen in recent non-relativistic Brueckner-Hartree-Fock calculations.

\section{The effective nuclear interaction in the spin channel}

In order to investigate this point more closely, we have tried a large variety of modelizations of the nuclear interaction commonly used to describe, on the one hand the properties of nuclei and of heavy-ion collisions, on the other hand the properties of neutron stars. Ideally, a single model should be able to accommodate all these facts before we can risk to perform extrapolations and make predictions in less accessible situations such as those encountered in protoneutron stars. Among the interactions probed were:

$$
\begin{aligned}
& - \text { The Skyrme interaction } \\
& \begin{aligned}
V(r)= & t_{0}\left(1+x_{0} P_{\sigma}\right) \delta(r) \\
& +\frac{1}{2} t_{1}\left(1+x_{1} P_{\sigma}\right)\left[k^{\prime 2} \delta(r)+\delta(r) k^{2}\right] \\
& +t_{2}\left(1+x_{2} P_{\sigma}\right) k^{\prime} \delta(r) k+\frac{1}{6} t_{3}\left(1+x_{3} P_{\sigma}\right) \rho_{N}^{\alpha} \delta(r) .
\end{aligned}
\end{aligned}
$$

The Skyrme interaction leads to a good description of properties of nuclei, nuclear matter and neutron stars. Recent parametrizations adapted to non-symmetric nuclear matter have been developed in the context of neutron stars, like the Skyrme-Lyon series [10] which was adjusted to reproduce microscopical neutron matter calculations. All Skyrme interactions (except the rather old and inaccurate SV parametrization [11]) lead to spin instabilities. 


\section{- The Gogny interaction}

It has been argued that the reason for this behavior of the Skyrme interaction could be that it is written only as a combination of contact interactions. On the other hand, the Gogny interaction consists of a finite range part, to which a Skyrme-like contact term is added to describe correlations:

$$
\begin{aligned}
V(r)= & \sum_{i=1,2}\left(t_{i}^{W}+t_{i}^{B} P_{\sigma}-t_{i}^{H} P_{\tau}-t_{i}^{M} P_{\sigma} P_{\tau}\right) \exp -\left(r / a_{i}\right)^{2} \\
& +\sum_{i=1,2} t_{3 i}\left(1+x_{3 i} P_{\sigma}\right) \rho^{\alpha_{i}} \delta(r) .
\end{aligned}
$$

The Gogny interaction yields a good description of the properties of nuclei and nuclear matter. Note that the original set D1S is not suitable for neutron matter, which would be found to be unstable and collapse. An improved parametrization $\mathrm{D} 1 \mathrm{P}$ has been developed by Farine et al. [12]: it is adjusted to reproduce microscopical neutron matter calculations. It is indeed found [6] that the spin instability, which in the Skyrme models was found to occur around 1.5-3 times the saturation density $n_{\text {sat }}$ (for some models, even below $n_{\text {sat }}$ !) is delayed in the D1P Gogny model to high densities, where other phenomena, like hyperon production and the transition to quark gluon plasma, are expected to occur anyway.

\section{- The Modified Seyler-Blanchard interaction}

In order to see whether the finite-range character of the interaction is the key to the suppression of spin instability, we next introduced several versions of the Modified Seyler-Blanchard interaction (MSB). This interaction, originally introduced by Myers and Swiatecki [13], consists of a finite-range (Yukawa) term and is momentum and density dependent:

$$
V=-C_{u l}\left[1-\frac{p^{2}}{b^{2}}-d^{2}\left(\rho_{1}+\rho_{2}\right)^{n}\right] \frac{e^{-r / a}}{r / a}
$$

with parameters $C_{u l}$ for $\{n \uparrow, n \downarrow, p \uparrow, p \downarrow\}$ It yields a good description of the properties of nuclei, nuclear matter and neutron stars. It leads to spin instabilities at about twice the saturation density, and indeed has been used to describe the consequences of having polarized nuclear matter in the context of neutron stars [14]. The results denoted below by "MSB" will use the same values of the parameters as taken in [14].

\section{- The density-dependent Michigan 3-range interaction}

An interesting alternative to the Gogny interaction is the density-dependent Michigan 3-range interaction (M3Y). Whereas the Gogny force use Gaussians in the finiterange part, the M3Y uses three Yukawas with ranges corresponding to Compton lengths of $\sigma, \omega$ and $\pi$ mesons. The phenomenological versions by Khoa et al. [15] or by Nakada [16] are based upon $G$-matrix elements of the Paris potential. The density dependence is introduced in 2 different ways: by multiplying the whole potential by a density-dependent scale factor $\mathcal{F}(\rho)$ [15], or by adding

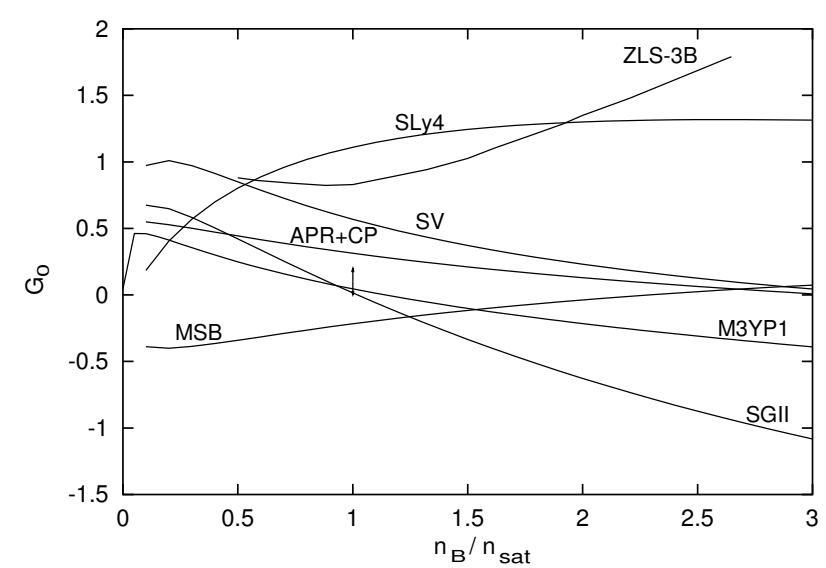

Fig. 1. Density dependence of spin Landau parameter $G_{0}$ in symmetric nuclear matter (meaning of the labels: see text).

a density dependent Skyrme-like contact interaction [16]. A good description of the properties of nuclei is thus obtained. This interaction has been used to study neutronrich nuclei and to describe low-energy nucleus-nucleus collisions [15]. For a study of the application of this model to the description of neutron star properties, see [17].

\section{- Parametrizations of microscopical calculations}

We will compare these results to parametrizations of recent microscopical calculations which were explicitly performed in view of investigating the spin stability of nuclear matter and of applications to the neutrino-nucleon crosssection in protoneutron stars. On the one hand, we have the Brueckner-Hartree-Fock calculation of polarized nuclear matter performed by Vidaña and Bombaci [18] and the extraction of Landau parameters in pure neutron matter with application to the neutrino-nucleon cross-section published by Margueron et al. [19] (denoted here by the label "MVB"). A similar work was done by Zuo et al. and Shen et al. [20] in symmetric nuclear matter (denoted here by the label "ZLS"). On the other hand, we used a parametrization of variational calculations by Akmal, Pandharipande and Ravenhall [21] for spin saturated systems, supplemented by an extension by Cowell and Pandharipande [22] for spin-polarized systems (denoted here by the label "APR $+\mathrm{CP}$ "). A notable feature of the variational calculation is present around twice the saturation density and interpreted by the authors of the calculation as a transition to a pion condensed phase.

\section{Density dependence of the Landau parameters}

The Landau parameters have been calculated for each model and their density dependence investigated [17]. A sample of these results is displayed in figs. 1-4.

A first test that the Landau parameters have to pass is to reproduce the experimental constraints in symmetric nuclear matter at saturation density. Such a constraint 


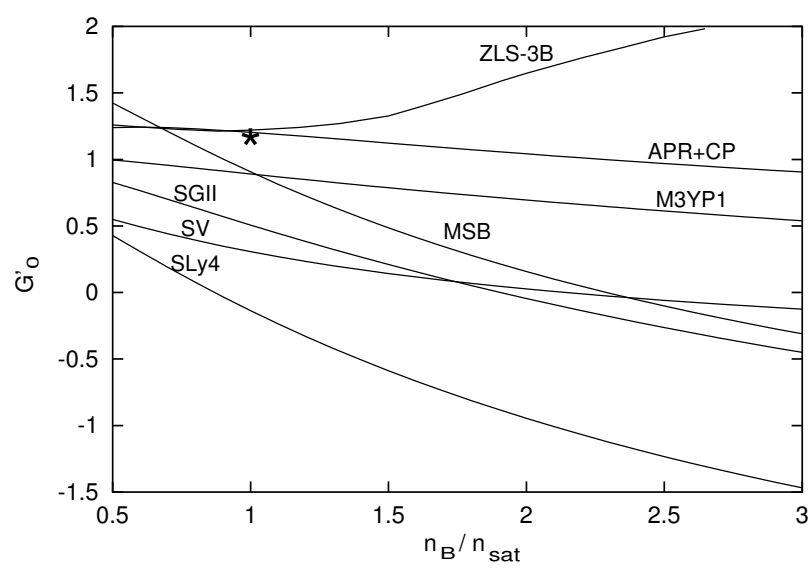

Fig. 2. Density dependence of spin-isospin Landau parameter $G_{0}^{\prime}$ in symmetric nuclear matter (labels: see text)

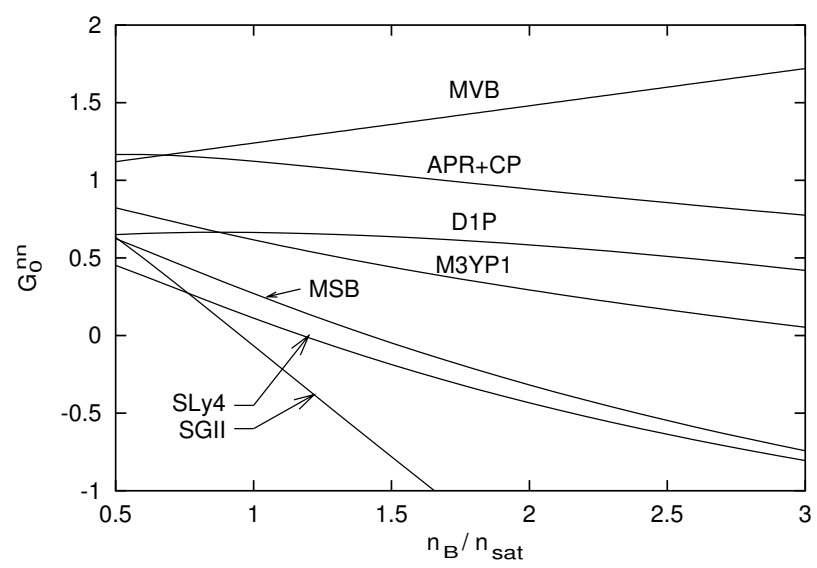

Fig. 3. Density dependence of spin Landau parameter $G_{0}^{n n}$ in pure neutron matter (labels: see text).

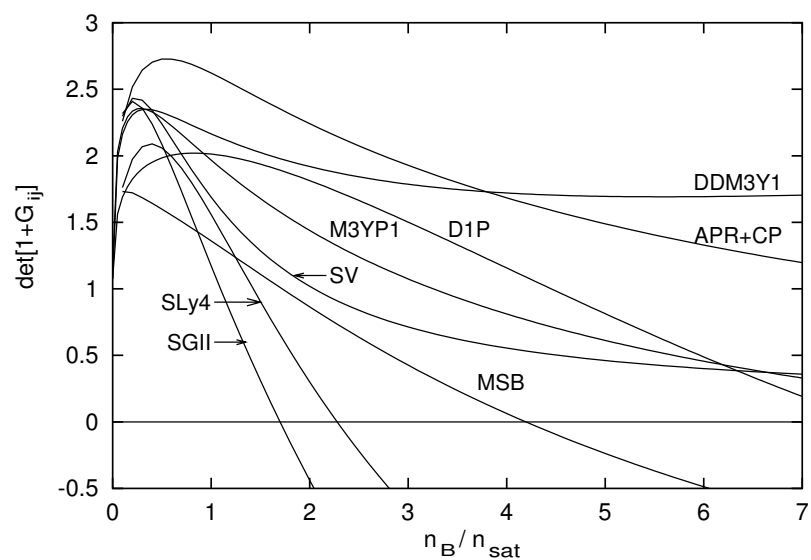

Fig. 4. Criterion for ferromagnetic instability in neutron star matter (labels: see text).

can be derived from the observation of the Gamow-Teller resonance in the spin-isospin channel. Zuo et al. [20] quote a value $G_{0}^{\prime} \simeq 1.18$ referring to a work by Suzuki [23] (indicated by a star in fig. 2). In an older work by Bäckman et al., [24] a higher value $G_{0}^{\prime} \simeq 1.6-1.7$ is quoted. In the spin channel, the absence of a clear resonance sets a constraint on the value of $G_{0}$, namely it is compatible with zero within some error bars. From [24] we will take $G_{0}=0.1 \pm 0.1$ (indicated by a double arrow in fig. 1).

- In the spin-isospin channel ("Gamow Teller")

The microscopical calculations (Brueckner-Hartree-Fock from ZLS and variational from APR $+\mathrm{CP}$ ) reproduce the experimental value at saturation density determined from observation of the Gamow-Teller giant resonance. They coincide for $n_{B}<n_{\text {sat }}$ but differ strongly at high density. The M3Y interactions are also compatible with the experimental value at saturation density. On the other hand, the Skyrme interactions do not generally reproduce the quoted value for $G_{0}^{\prime}$. An exception should be made for the SGII parametrization [25] of the Skyrme force, which was specially adjusted to reproduce the Gamow-Teller resonance. The value of $G_{0}^{\prime}$ obtained for this model is nevertheless rather low. A further discussion of the adequacy of the Skyrme force to describe the spin-isospin modes can be found in Bender et al. [26].

\section{- In the spin channel ("Fermi")}

The parametrization of variational results APR $+\mathrm{CP}$ also reproduces the recommended value $G_{0}=0.1 \pm 0.1$. The Landau parameters extracted from BHF calculations in contrast obtain too high values. The Skyrme results are in general too high at saturation density, except, again, the SGII parametrization. The MSB result is compatible with the available data. Finally, the M3Y models are again compatible with the experimental constraint.

\section{- Pure neutron matter}

In pure neutron matter there is only one relevant Landau parameter, $G_{0}^{n n}$, which is displayed in fig. 3. Its behavior is not very different from that of $G_{0}^{\prime}$ in symmetric nuclear matter. Let us note that the SGII parametrization leads to an instability at a low value of the density $\sim 1.6 n_{\text {sat }}$ in pure neutron matter.

\section{- Neutron star matter in $\beta$ equilibrium}

In neutron star matter in $\beta$ equilibrium there are three different Landau parameters, $G_{0}^{n n}, G_{0}^{p p}$ and $G_{0}^{n p}$. They can be combined to form the criterion for spin instability as explained in sect. 2.2. A sample of the results is displayed in fig. 4. We can see that the Skyrme parametrizations indeed lead to an instability at low density. In particular, this is also the case of the SGII parametrization: even though it was adjusted to give optimal results in symmetric nuclear matter, the stability criterion is here dominated by the interaction between neutrons. The MSB model presents a transition around $4 n_{\text {sat }}$, while the other models are stable up to at least $7 n_{\text {sat }}$.

\section{Response functions}

Parametrizations which are stable with respect to spin fluctuations give rise to response functions such as those 


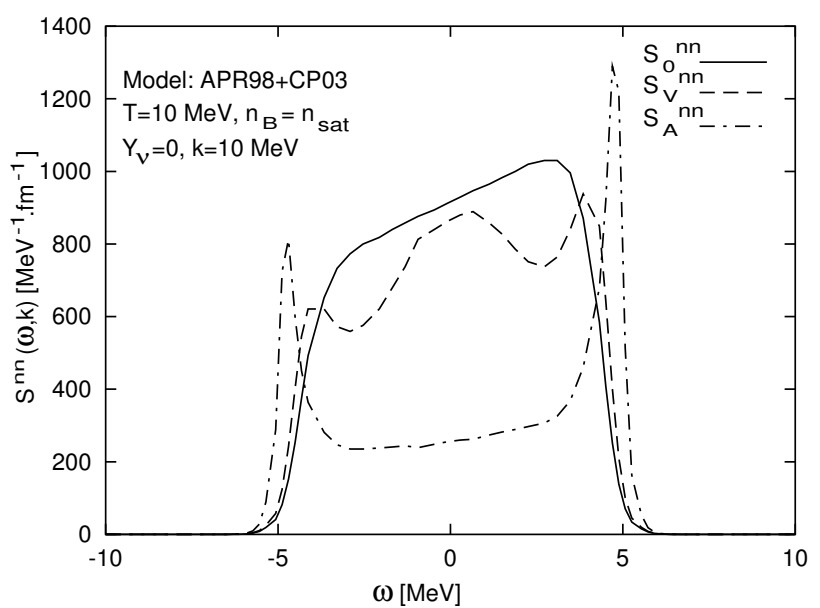

Fig. 5. Response function for the APR $+\mathrm{CP}$ model in neutron star matter in $\beta$ equilibrium.

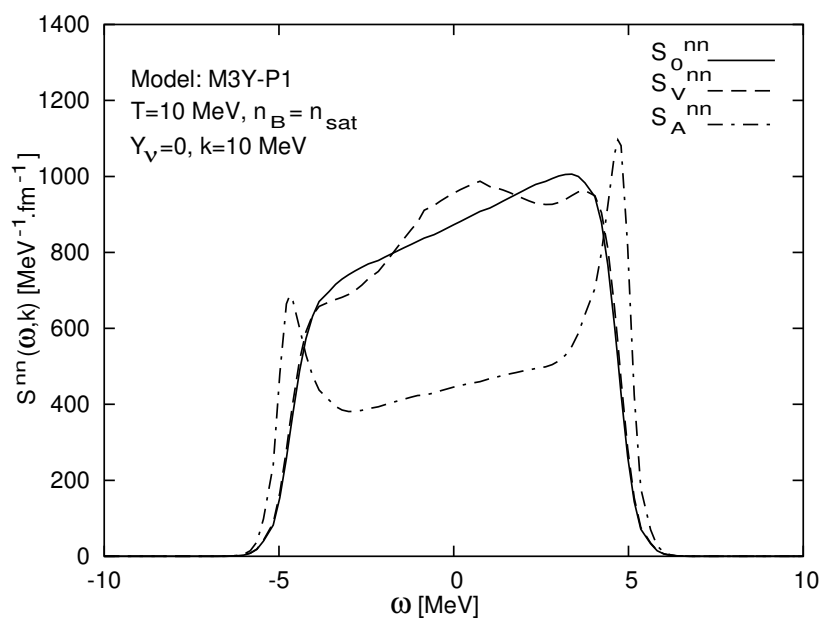

Fig. 6. Response function for the M3YP1 model in neutron star matter in $\beta$ equilibrium.

displayed in figs. 5 and 6. This includes microscopical calculations (variational or Brueckner), the M3Y models as well as the Gogny parametrization at low density. In these figures, we have represented the contribution of the neutrons to the response function for the APR $+\mathrm{CP}[21$, 22] (fig. 5) and M3YP1 [16] (fig. 6) parametrizations at saturation density, for neutrino-free matter in $\beta$ equilibrium, at a temperature $T=10 \mathrm{MeV}$ and for a momentum transfer $k=10 \mathrm{MeV}$. The full line corresponds to the response $S_{0}$ in the mean-field approximation. Also represented are the vector $S_{V}$ (dashed line) and axial $S_{A}$ (dash-dotted line) response functions in the random phase approximation. It is seen that the RPA response for small energy transfer is reduced with respect to the mean-field approximation, in agreement with previous findings. The vector response displays a zero sound mode and the axial response a spin-zero sound mode, both appearing at the high energy edge of the strength distribution. The contribution of these modes to the neutrino-nucleon cross-section is strongly suppressed by phase-space as

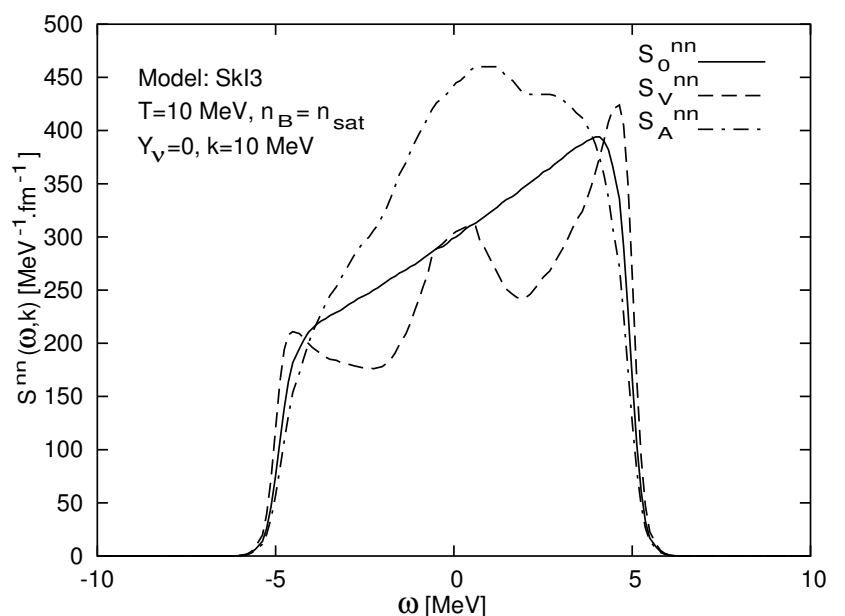

Fig. 7. Response function for the SkI3 Skyrme model in neutron star matter in $\beta$ equilibrium.

well as by Pauli-blocking factors. The global effect will be a reduction of the neutrino-nucleon cross-section with respect to the mean-field approximation.

On the other hand, parametrizations which lead to a spin instability will give rise to response functions such as those displayed in fig. 7. This is the case of the Skyrme or MSB interactions as well as of the Gogny interaction at high density. The model chosen for fig. 7 is the SkI3 Skyrme parametrization, which fulfills the criterion for spin instability for a density $\sim 3.1 n_{\text {sat }}$, whereas the spin responses are plotted, as in figs. 5-6, at saturation density: even though we are still far from the transition, a precursor of the pole is already to be seen as an enhancement in the axial response function at zero energy $\omega=0$. At higher densities, the response is completely dominated by the development of the instability.

\section{Mean free path}

Finally we represent in fig. 8 the RPA correction to the cross-section for various models as a function of density, i.e. we plotted the ratio of the total cross-sections (or equivalently the ratio of mean free paths) in the meanfield and random phase approximations $\sigma_{\mathrm{RPA}} / \sigma_{\mathrm{MF}}=$ $\lambda_{\mathrm{MF}} / \lambda_{\mathrm{RPA}}$.

Again we see that Skyrme models (all except SV) or Modified Seyler-Blanchard (MSB) display an enhancement of the cross-section in the RPA with respect to the mean-field calculation and a divergence in the vicinity of spin instability. The Skyrme SV interaction, which is stable against spin excitations, still displays an enhancement (by a factor of 2 at $3 n_{\text {sat }}$ )

The microscopical models (BHF or variational) predict a reduction of the cross-section from RPA correlations: at $3 n_{\text {sat }}$, by a factor of 2 for ZLS, a factor of 3 for APR + CP, by a factor of 4 for MVB. A caveat is in order for model APR + CP: The parametrization performed by Cowell and Pandharipande [22] is only available up to $n_{B}=0.24 \mathrm{fm}^{-3}$, i.e. below the transition observed 


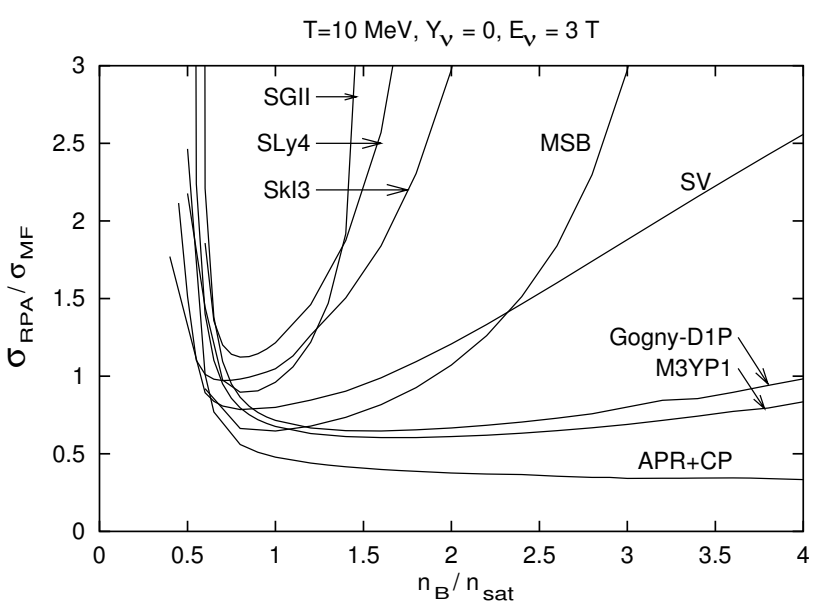

Fig. 8. RPA correction to the cross-section for various models of the nuclear interaction in neutron star matter in $\beta$ equilibrium (labels: see text).

by Akmal et al. [21] around $\sim 2 n_{\text {sat }}$ and interpreted as pion condensation. It will be important to investigate the role of tensor force [27] (which was not taken into account here) in relation with the consequences of this transition on the neutrino mean free path.

The density-dependent Michigan three range models also predict a reduction of the cross-section from RPA corrections, but by a smaller factor (up to $40 \%$ ). Very similar results are obtained for 8 different parametrizations of the M3Y forces. More detailed results for the M3Y forces will be published in [17].

\section{Conclusion and outlook}

The object of this contribution has been to investigate whether our knowledge of the nuclear interaction in the spin $S=1$ is established on a sufficiently firm basis to allow to make predictions for the neutrino-nucleon crosssection in protoneutron star matter, and eventually to predict the shape of the neutrino burst. The answer is, unfortunately, not positive at the present time. This should not in fact come as a surprise. The interaction, which we have characterized here in terms of the Landau parameters, is immediately translatable in terms of "spin asymmetry" $E_{\mathrm{s}}$ and "spin-isospin asymmetry" $E_{\mathrm{st}}$ energies. A similar uncertainty as to the behavior of the asymmetry energy $E_{\mathrm{t}}$ (or, equivalently, of the Landau parameter $F_{0}^{\prime}$ ) as a function of density has long been the subject of intense research. Experiments involving neutron-rich nuclei or neutrino-nucleon scattering, in parallel with renewed efforts of groups who perform Brueckner as well as variational many-body calculations in the spin sector, will hopefully help reduce this uncertainty. In the meantime, some care has to be exerted when choosing an interaction to describe the neutrino-nucleon cross-section.

While the microscopical calculations are - up to a certain point - parameter free and more satisfactory from a conceptual point of view, their use is hampered by the heavy numerical work involved, so that a parametrization seems necessary. On the other hand, we have seen that the phenomenological interactions are not well under control. One could of course extract the Landau parameters from the microscopical calculations and parametrize them. The M3Y interactions can be a valuable alternative, since they have the interesting properties of reproducing without special adjustments the spin Landau parameters at saturation, and of being free of spin instabilities. Moreover, the 3-range Yukawa structure allow a more transparent connection to the meson-exchange models of the nucleon-nucleon potential: there have indeed been attempts to parametrize Brueckner calculations in terms of M3Y interactions [28].

This work was supported by the Spanish-European (FICYT/FEDER) grant No. PB02-076.

\section{References}

1. T. Janka, Astron. Astrophys. 368, 527 (2001).

2. J. Pons et al., Astrophys. J. 513, 780 (1999).

3. N. Iwamoto, C.J. Pethick, Phys. Rev. D 25, 313 (1982).

4. S. Reddy et al., Phys. Rev. C 59, 2888 (1999).

5. J. Navarro, E.S. Hernandez, D. Vautherin, Phys. Rev. C 60, 045801 (1999).

6. J. Margueron, PhD Thesis, Orsay University, France (2001).

7. L. Mornas, Eur. Phys. J. A 23, 365 (2005).

8. See, e.g.: A. Vidaurre, J. Navarro, J. Bernabeu, Astron. Astrophys. 135, 361 (1984); M. Kutschera, W. Wojcik, Phys. Lett. B 325, 271 (1994); J. Margueron, J. Navarro, Nguyen Van Giai, Phys. Rev. C 66, 014303 (2002); A.A. Isayev, JETP 77, 251 (2003); A.A. Isayev, J. Yang, Phys. Rev. C 69, 025801 (2004).

9. P. Bernardos et al., Phys. Lett. B 356175 (1995); T. Maruyama, T. Tatsumi, Nucl. Phys. A 693, 710 (2001); 721, 265c (2003).

10. E. Chabanat et al., Nucl. Phys. A 635, 231 (1998).

11. M. Beiner et al., Nucl. Phys. A 238, 29 (1975).

12. M. Farine et al., J. Phys. G 25, 863 (1999).

13. W.D. Myers, W.J. Swiatecki, Ann. Phys. (N.Y.) 55, 395 (1969); 204, 401 (1990).

14. V.S. Uma Maheswari et al., Nucl. Phys. A 615, 516 (1997); V.S. Uma Maheswari, J.N. De, S.K. Samaddar, Phys. Rev. D 57, 3255 (1998).

15. D.T. Khoa, W. von Oertzen, A.A. Ogloblin, Nucl. Phys. A 602, 98 (1996).

16. H. Nakada, Phys. Rev. C 68, 014316 (2003).

17. L. Mornas, in preparation.

18. I. Vidaña, I. Bombaci, Phys. Rev. C 66, 045801 (2002).

19. J. Margueron, I. Vidaña, I. Bombaci, Phys. Rev. C 68, 055806 (2003).

20. W. Zuo, C. Shen, N. Van Giai, Phys. Rev. C 67, 037301 (2003); C. Shen et al., Phys. Rev. C 68, 055802 (2003).

21. A. Akmal, V.R. Pandharipande, D.G. Ravenhall, Phys. Rev. C 58, 1804 (1998).

22. S.T. Cowell, V.R. Pandharipande, Phys. Rev. C 67, 035504 (2003). 
23. T. Suzuki, H. Sakai, Phys. Lett. B 455, 25 (1999); A. Kurasawa, T. Suzuki, N. Van Giai, Nucl. Phys. A 731, 114 (2004).

24. S.O. Bäckmann, G.E. Brown, J.A. Niskanen, Phys. Rep. 124, 1 (1985).

25. N. Van Giai, H. Sagawa, Phys. Lett. B 106, 379 (1981).
26. M. Bender et al., Phys. Rev. C 65, 054322 (2002).

27. E. Olsson, C.J. Pethick, P. Haensel, Phys. Rev. C 70 $025804(2004)$.

28. F. Hofmann, H. Lenske, Phys. Rev. C 57, 2281 (1998); G. Bartnitzky et al., Phys. Lett. B 386, 7 (1996). 